\title{
The order dimension of Bruhat order on infinite Coxeter groups
}

\author{
Nathan Reading* \\ Mathematics Department \\ University of Michigan \\ Ann Arbor, MI 48109, USA \\ nreading@umich.edu \\ Debra J. Waugh \\ Division of Mathematics and Computer Science \\ Alfred University \\ Alfred, NY 14802, USA \\ djwaugh@verizon.net
}

Submitted: Sep 27, 2004; Accepted: Jan 11, 2005; Published: Feb 14, 2005

2000 Mathematics Subject Classifications: 20F55; 06A07

\begin{abstract}
We give a quadratic lower bound and a cubic upper bound on the order dimension of the Bruhat (or strong) ordering of the affine Coxeter group $\tilde{A}_{n}$. We also demonstrate that the order dimension of the Bruhat order is infinite for a large class of Coxeter groups.
\end{abstract}

\section{Introduction}

We study the order dimension of the Bruhat (or strong) ordering on finitely generated infinite Coxeter groups. In particular for the affine group $\tilde{A}_{n}$, we prove the following:

Theorem 1.1. The order dimension of the Bruhat ordering of the Coxeter group $\tilde{A}_{n}$ satisfies the following bounds:

$$
n(n+1) \leq \operatorname{dim}\left(\tilde{A}_{n}\right) \leq(n+1)\left\lfloor\frac{(n+1)^{2}}{4}\right\rfloor .
$$

\footnotetext{
*Partially supported by NSF grant DMS-0202430.
} 
In particular $\operatorname{dim}\left(\tilde{A}_{1}\right)=2$ and $\operatorname{dim}\left(\tilde{A}_{2}\right)=6$, but exact values are unknown for $n \geq 3$.

The bounds of Theorem 1.1 arise from the following theorem, the finite case of which is $[14$, Theorem 6$]$.

Theorem 1.2. If $P$ is a finitary poset of finite or countable cardinality, then

$$
\operatorname{width}(\operatorname{Dis}(P)) \leq \operatorname{dim}(P) \leq \operatorname{width}(\operatorname{Irr}(P)) .
$$

A poset is finitary if every principal order ideal is finite. The posets $\operatorname{Dis}(P)$ and $\operatorname{Irr}(P)$ are the subposets of $P$ consisting respectively of dissectors and join-irreducibles (see Section 2). Bruhat orders are finitary, so Theorem 1.2 applies. We prove the lower bound in Theorem 1.1 by exhibiting an antichain of dissectors in $\tilde{A}_{n}$ and prove the upper bound by exhibiting a decomposition of $\operatorname{Irr}\left(\tilde{A}_{n}\right)$ into chains. The proof of the lower bound employs the combinatorics of reduced words and the affine permutations defined by Lusztig [12]. The decomposition into chains uses geometric methods, particularly the following theorem, which is a special case of [19, Theorem 4.8] (see also [19, Corollary $4.13])$.

Theorem 1.3. [Stembridge] Let $\widetilde{W}$ be an affine Coxeter group with Weyl group $W$. Let ${ }^{J} \widetilde{W}^{K}$ be a minuscule two-sided quotient of $\widetilde{W}$. Then Bruhat order on ${ }^{J} \widetilde{W}^{K}$ is isomorphic to a connected component of the standard order on dominant weights for a root system associated to $W$.

The quotient ${ }^{J} \widetilde{W}^{K}$ is minuscule if both $\widetilde{W}_{J}$ and $\widetilde{W}_{K}$ are isomorphic to $W$. When $\widetilde{W}$ is $\tilde{A}_{n}$, every maximal parabolic subgroup is isomorphic to $W=A_{n}$. Theorem 1.3 implies an upper bound of $n(n+1)^{2}$ on the order dimension, and makes it possible to identify the join-irreducibles and obtain the improved upper bound of Theorem 1.1. Computer calculations suggest that $n(n+1)$ is in fact the width of $\operatorname{Dis}\left(\tilde{A}_{n}\right)$ and that $(n+1)\left\lfloor\frac{(n+1)^{2}}{4}\right\rfloor$ is the width of $\operatorname{Irr}\left(\tilde{A}_{n}\right)$, so the bounds cannot be sharpened using Theorem 1.2.

Let $K$ be such that $A_{n}$ is the maximal parabolic subgroup $\left(\tilde{A}_{n}\right)_{K}$. The chain decomposition of $\operatorname{Irr}\left(\tilde{A}_{n}\right)$, given in Section 9, restricts to a chain decomposition of $\operatorname{Irr}\left(\tilde{A}_{n}^{K}\right)$ which gives an upper bound of $\left\lfloor\frac{(n+1)^{2}}{4}\right\rfloor$ on the order dimension of $\tilde{A}_{n}^{K}$. Proposition 9.1 records the following fact, which was pointed out by Stembridge [20]: For any affine Coxeter group $\widetilde{W}$, if $(\widetilde{W})_{K}$ is the associated Weyl group $W$ then the Bruhat order on $\widetilde{W}^{K}$ contains an interval isomorphic to the Bruhat order on $W$. Thus in particular, the order dimension of the Bruhat order on $\tilde{A}_{n}^{K}$ is greater than or equal to the order dimension of the Bruhat order on $A_{n}$. In [14], the order dimension of the Bruhat order on $A_{n}$ is determined to be $\left\lfloor\frac{(n+1)^{2}}{4}\right\rfloor$, which is therefore equal to the order dimension of the Bruhat order on $\tilde{A}_{n}^{K}$.

We show (Proposition 5.1) that rigid elements are dissectors, and apply Theorem 1.2 to exhibit an infinite class of Coxeter groups each of which has infinite order dimension. In the process, we classify the Coxeter groups for which the number of rigid elements of length $l$ is an unbounded function of $l$ (Proposition 5.2).

The organization of this paper is as follows: Definitions and results on finitary posets are found in Section 2, followed in Section 3 by background on order quotients. Section 4 
gives background on Bruhat order. Section 5 identifies an infinite class of Coxeter groups each of which has infinite-dimensional Bruhat order. Section 6 describes the realization of $\tilde{A}_{n}$ by affine permutations, leading to the proof in Section 7 of the lower bound of Theorem 1.1. In Section 8, we describe the standard order on dominant weights and identify the join-irreducibles of the connected components of the standard order on dominant weights. Section 9 is the proof of the upper bound of Theorem 1.1.

\section{$2 \quad$ Finitary posets}

We begin by establishing notation, definitions, and general tools related to finitary posets. An order ideal in a poset $P$ is a set $I$ such that $x \in I$ and $y \leq x$ implies $y \in I$. Given $x \in P$, define

$$
\begin{aligned}
D(x) & :=\{y \in P: y<x\} \\
U(x) & :=\{y \in P: y>x\} \\
D[x] & :=\{y \in P: y \leq x\} \\
U[x] & :=\{y \in P: y \geq x\} .
\end{aligned}
$$

An order ideal of the form $D[x]$ for some $x \in P$ is called a principal order ideal. A poset $P$ is called finitary if every principal order ideal has a finite number of elements. This definition is consistent with the definition of finitary distributive lattices in [16, Section 3.4]. Only finitary posets are considered in this paper.

The order dimension $\operatorname{dim}(P)$ of a finitary poset $P$ is the smallest cardinal $d$ such that $P$ is the intersection of $d$ linear extensions of $P$. Equivalently, the order dimension is the smallest $d$ so that $P$ can be embedded as a subposet of $\mathbb{R}^{d}$ with componentwise partial order. A simple construction shows that the order dimension of any poset is at most its cardinality. In this paper, we do not consider any posets whose cardinality is more than countably infinite. The standard example of a poset of dimension $n$ is the set of subsets of $[n]:=\{1,2, \ldots n\}$ of cardinality 1 or $n-1$, ordered by inclusion. For more information on order dimension, see [21].

Given $x$ and $y$, if $U[x] \cap U[y]$ has a unique minimal element, this element is called the join of $x$ and $y$ and is written $x \vee^{P} y$ or simply $x \vee y$. If $D[x] \cap D[y]$ has a unique maximal element, it is called the meet of $x$ and $y, x \wedge_{P} y$ or $x \wedge y$. The notation, $x \vee y=a$ means " $x$ and $y$ have a join, which is $a$," and similarly for other statements about joins and meets. Given a set $S \subseteq P$, if $\cap_{x \in S} U[x]$ has a unique minimal element, it is called $\vee S$. The join $\vee \emptyset$ is $\hat{0}$ if $P$ has a unique minimal element $\hat{0}$, and otherwise $\vee \emptyset$ does not exist. If $\cap_{x \in S} D[x]$ has a unique maximal element, it is called $\wedge S$. The meet $\wedge \emptyset$ exists if and only if a unique maximal element $\hat{1}$ exists, in which case they coincide. A poset is called a lattice if every finite set has a join and a meet.

An element $a$ of a poset $P$ is join-irreducible if there is no set $X \subseteq P$ with $a \notin X$ and $a=\vee X$. When $P$ is finitary, this can be rephrased: $a$ is join irreducible if there is no finite set $X \subseteq P$ with $a \notin X$ and $a=\vee X$. If $P$ has a unique minimal element $\hat{0}$, then $\hat{0}$ is $\vee \emptyset$ and thus is not join-irreducible. In a lattice, $a$ is join-irreducible if and only if it covers 
exactly one element. Such an element is also join-irreducible in a non-lattice $P$, but if the set $C$ of elements covered by some $a \in P$ has $|C|>1$ then $a$ is join-irreducible if and only if $C$ has an upper bound incomparable to $a$. A minimal element of a non-lattice is also join-irreducible, if it is not $\hat{0}$. If $x \in P$ is not join-irreducible, then $x=\vee D(x)$. The subposet of $P$ induced by the join-irreducible elements is denoted $\operatorname{Irr}(P)$. An element $a$ of a poset $P$ is meet-irreducible if there is no set $X \subseteq P$ with $a \notin X$ and $a=\wedge X$.

For $x \in P$, let $I_{x}$ denote $D[x] \cap \operatorname{Irr}(P)$, the set of join-irreducibles weakly below $x$ in $P$. The following proposition restricted to the case of finite posets is [14, Proposition 9]. The proof holds for finitary posets without alteration.

Proposition 2.1. Let $P$ be a finitary poset, and let $x \in P$. Then $x=\vee I_{x}$.

A poset is called directed if for every $x, y \in P$, there is some $z \in P$ with $z \geq x$ and $z \geq y$. An element $x$ in a finitary poset $P$ is called a dissector of $P$ if $P-U[x]$ is nonempty and directed. Call $x$ a strong dissector if $P-U[x]=D[\beta(x)]$ for some $\beta(x) \in P$. In other words, $P$ can be dissected as a disjoint union of the principal order filter generated by $x$ and the principal order ideal generated by $\beta(x)$. A strong dissector is a dissector, and if $P$ is finite then the two notions are equivalent. The subposet of dissectors of $P$ is called $\operatorname{Dis}(P)$. In the lattice case the definition of dissector coincides with the notion of a prime element. An element $x$ of a lattice $L$ is called prime if whenever $x \leq \vee Y$ for some $Y \subseteq L$, then there exists a $y \in Y$ with $x \leq y$. The following easy proposition, proven in [11] for finite posets, holds for finitary posets by the same proof.

Proposition 2.2. If $x$ is a dissector then $x$ is join-irreducible.

The converse is not true in general. A poset $P$ in which every join-irreducible is a dissector is called a dissective poset. In [11] this property of a finite poset is called "clivage."

We now prove Theorem 1.2 by a straightforward modification of the proof of the finite case $[14$, Theorem 6].

Proof of Theorem 1.2. If $\operatorname{Irr}(P)$ has infinite width, then the upper bound is immediate. Otherwise let $C_{1}, C_{2}, \ldots, C_{d}$ be a chain decomposition of $\operatorname{Irr}(P)$. For each $m \in[d]$ and $x \in P$, let $f_{m}(x):=\left|I_{x} \cap C_{m}\right|$. By Proposition 2.1, $x \leq y$ if and only if $I_{x} \subseteq I_{y}$ if and only if $f_{m}(x) \leq f_{m}(y)$ for every $m \in[d]$. Thus $x \mapsto\left(f_{1}(x), f_{2}(x), \ldots, f_{d}(x)\right)$ is an embedding of $P$ into $\mathbb{N}^{d}$.

For the lower bound, consider a finite antichain $A$ in $\operatorname{Dis}(P)$. For each $a \in A$, define $b(a)$ to be be an upper bound in $P-U_{P}[a]$ for the set $A-\{a\}$. A finite number of applications of the property that $a$ is a dissector assures the existence of such an element. The subposet of $P$ induced by $A \cup b(A)$ is isomorphic to the standard example of a poset of dimension $|A|$. Thus $\operatorname{dim}(P) \geq \operatorname{dim}(A \cup b(A))=|A|$. If the width of $\operatorname{Dis}(P)$ is finite, choose $A$ to be a largest antichain. If the width is countable, then consider a sequence of antichains whose cardinality approaches infinity.

Corollary 2.3. If $P$ is a finitary dissective poset such that width $(\operatorname{Irr}(P))$ is finite or countable, then $\operatorname{dim}(P)=$ width $(\operatorname{Irr}(P))$. 
The dissective property is a generalization of the distributive property, in the following sense:

Proposition 2.4. A finitary lattice $L$ is distributive if and only if it is dissective.

Proposition 2.4 is well known $[8,13]$ in the finite case with different terminology, and the proof in the finitary case is a straightforward generalization.

The Bruhat order on the finite Coxeter groups of types $\mathrm{A}, \mathrm{B}$ and $\mathrm{H}$ is known to be dissective [14]. The Bruhat order on $\tilde{A}_{1}$ is easily verified to be dissective. Proposition 4.6 implies that the Bruhat order on a Coxeter group is dissective if and only if each of its maximal double quotients is dissective. The standard order on the dominant weights of $A_{2}$ is a distributive lattice [18, Theorem 3.3], and thus by Theorem 1.3, the Bruhat order on $\tilde{A}_{2}$ is dissective. This is reflected in the fact that the upper and lower bounds of Theorem 1.1 agree for $n=1$ and $n=2$. For $n>2$, the Bruhat order on $\tilde{A}_{n}$ is not dissective, because the standard order on the dominant weights of $A_{n}$ is a non-distributive lattice [18, Theorem 3.2].

\section{Order Quotients}

In this section, we define poset congruences and order quotients and relate them to joinirreducibles and dissectors. The results in this section are generalizations to the infinite case of results from [14]. For more information on poset congruences and order quotients see $[5,14,15]$. Let $P$ be a finitary poset with an equivalence relation $\Theta$ defined on the elements of $P$. Given $a \in P$, let $[a]_{\Theta}$ denote the $\Theta$-equivalence class of $a$.

Definition 3.1. The equivalence relation $\Theta$ is a congruence if:

(a) Every equivalence class has a unique minimal element.

(b) The projection $\pi_{\downarrow}: P \rightarrow P$, mapping each element a of $P$ to the minimal element in $[a]_{\Theta}$, is order-preserving.

(c) Whenever $\pi_{\downarrow} a \leq b$, there exists $t \in[b]_{\Theta}$ such that $a \leq t$ and $b \leq t$.

Chajda and Snášel [5, Definition 2] give a version of Definition 3.1 holding for arbitrary posets and show that their definition is equivalent to lattice congruence when $P$ is a lattice.

Define a partial order on the congruence classes by $[a]_{\Theta} \leq[b]_{\Theta}$ if and only if there exist $x \in[a]_{\Theta}$ and $y \in[b]_{\Theta}$ such that $x \leq_{P} y$. The set of congruence classes under this partial order is $P / \Theta$, the quotient of $P$ with respect to $\Theta$. When $P$ is finitary, it is convenient to identify $P / \Theta$ with the induced subposet $Q:=\pi_{\downarrow} P$, as is typically done for example with quotients of Bruhat order. Such a subposet $Q$ is called an order quotient of $P$.

The finite cases of the following statements are [14, Propositions 26 and 27].

Lemma 3.2. Suppose $Q$ is an order quotient of a finitary poset $P$. If $x=\vee^{Q} Y$ for some $Y \subseteq Q$, then $x=\vee^{P} Y$. If $x=\vee^{P} Y$ for some $Y \subseteq P$, then $\pi_{\downarrow} x=\vee^{Q}\left(\pi_{\downarrow} Y\right)$. 
Proof. Suppose $x=\vee^{Q} Y$ for $Y \subseteq Q$ and suppose $z \in P$ has $z \geq y$ for every $y \in Y$. Then $\pi_{\downarrow} z \geq \pi_{\downarrow} y=y$ for every $y \in Y$, so $z \geq \pi_{\downarrow} z \geq x$. Thus $x=\vee^{P} Y$.

Suppose $x=\vee^{P} Y$ for $Y \subseteq P$. Then $\pi_{\downarrow} x \geq \pi_{\downarrow} y$ for every $y \in Y$. If there is some other $z \in Q$ with $z \geq \pi_{\downarrow} y$ for every $y \in Y$, then by condition (c) in Definition 3.1, for each $y \in Y$, there exists a $z_{y} \in[z]_{\Theta}$ such that $z_{y} \geq z$ and $z_{y} \geq y$. Since each $z_{y}$ has $z_{y} \geq \pi_{\downarrow} z=z$, by iterating condition (c), we obtain an element $z^{\prime}$, congruent to $z$, which is an upper bound for the set $\left\{z_{y}: y \in Y\right\}$. Since $P$ is finitary, $Y$ is a finite set, so we only have to iterate condition (c) a finite number of times. We have $z^{\prime} \geq y$ for every $y \in Y$, and so $z^{\prime} \geq x$. Thus also $\pi_{\downarrow}\left(z^{\prime}\right) \geq \pi_{\downarrow} x$, but $\pi_{\downarrow}\left(z^{\prime}\right)=z$, and so $\pi_{\downarrow} x=\vee^{Q}\left(\pi_{\downarrow} Y\right)$.

Proposition 3.3. Suppose $Q$ is an order quotient of a finitary poset $P$ and let $x \in Q$. Then $x$ is join-irreducible in $Q$ if and only if it is join-irreducible in $P$, and $x$ is a dissector of $Q$ if and only if it is a dissector of $P$. In other words,

$$
\begin{aligned}
\operatorname{Irr}(Q) & =\operatorname{Irr}(P) \cap Q \text { and } \\
\operatorname{Dis}(Q) & =\operatorname{Dis}(P) \cap Q .
\end{aligned}
$$

Proof. Suppose $x \in Q$ is join-irreducible in $Q$, and suppose $x=\vee^{P} Y$ for some $Y \subseteq P$. Then by Lemma 3.2, $x=\pi_{\downarrow} x=\vee^{Q}\left(\pi_{\downarrow} Y\right)$. Since $x$ is join-irreducible in $Q$, we have $x \in \pi_{\downarrow} Y$, and thus there exists an $x^{\prime} \in Y$ with $\pi_{\downarrow}\left(x^{\prime}\right)=x$ and in particular $x \leq x^{\prime}$. But since $x=\vee^{P} Y$, we have $x^{\prime} \leq x$ and so $x=x^{\prime} \in Y$. Conversely, suppose $x \in Q$ is join-irreducible in $P$, and suppose $x=\vee^{Q} Y$ for some $Y \subseteq Q$. Then by Lemma 3.2, $x=\vee^{P} Y$, so $x \in Y$. Thus $x$ is join-irreducible in $Q$.

Suppose $x \in Q$ is a dissector of $Q$, and let $y, z \in P-U_{P}[x]$. We need to find an upper bound in $P-U_{P}[x]$ for $y$ and $z$. Since $y \geq x, \pi_{\downarrow} y \geq x$, and similarly $\pi_{\downarrow} z \nsupseteq x$. Because $x$ is a dissector in $Q$, there is some $b \in Q-U_{Q}[x]$ with $b \geq \pi_{\downarrow} y$ and $b \geq \pi_{\downarrow} z$. By condition (c), there is an element $b^{\prime} \in P$, congruent to $b$, with $b^{\prime} \geq y$ and $b^{\prime} \geq b$. Again, by condition (c), there is an element $b^{\prime \prime}$ congruent to $b^{\prime}$ with $b^{\prime \prime} \geq z$ and $b^{\prime \prime} \geq b^{\prime}$. Thus $b^{\prime \prime}$ is an upper bound for $y$ and $z$, and since $b^{\prime \prime}$ is congruent to $b$, it is not in $U_{P}[x]$; if we did have $b^{\prime \prime} \geq x$, then we would have $b=\pi_{\downarrow}\left(b^{\prime \prime}\right) \geq \pi_{\downarrow} x=x$.

Conversely, suppose $x \in Q$ is a dissector of $P$, and let $y, z \in Q-U_{Q}[x]$. Thus also $y, z \in P-U_{P}[x]$, so there is some $b \in P-U_{P}[x]$ such that $b \geq y$ and $b \geq z$. Then $\pi_{\downarrow} b \geq \pi_{\downarrow} y=y$ and $\pi_{\downarrow} b \geq \pi_{\downarrow} z=z$. Since $b \geq \pi_{\downarrow} b$ and $b \nsupseteq x$, necessarily $\pi_{\downarrow} b \nsupseteq x$. In particular there is an upper bound $\pi_{\downarrow} b$ for $y$ and $z$ in $U_{Q}[x]$. Thus $x$ is a dissector in $P$.

\section{Bruhat Order on a Coxeter Group}

In this section we present background on Coxeter groups and on the Bruhat order. We study join-irreducibles and dissectors of Coxeter groups under the Bruhat order. For more details, and for proofs of results quoted here, see $[4,10]$.

A Coxeter group is a group $W$ given by a set $S$ of generators together with relations $s^{2}=1$ for all $s \in S$ and the braid relations $(s t)^{m(s, t)}=1$ for all $s \neq t \in S$. Each $m(s, t)$ is 
an integer greater than 1 , or is $\infty$. In the latter case no relation of the form $(s t)^{m}=1$ is imposed. The Coxeter group can be specified by its graph $\Gamma$, whose vertex set is $S$, with unlabeled edges whenever $m(s, t)=2$ and edges labeled $m(s, t)$ whenever $m(s, t)>3$. The graph is called simply laced if it has no labeled edges. A Coxeter group is irreducible if and only its graph is connected.

Important examples of Coxeter groups include the finite and affine Weyl groups. In this paper, we consider the affine Weyl group $\tilde{A}_{n}$ with $S=\left\{s_{0}, s_{1}, \ldots, s_{n}\right\}, m\left(s_{0}, s_{n}\right)=3$, $m\left(s_{i-1}, s_{i}\right)=3$ for $i \in[n]$ and $m=2$ otherwise. To simplify notation, subscripts are interpreted $\bmod n+1$, so that for example, $s_{n+1}=s_{0}$. Also, set $\langle i\rangle:=S-\left\{s_{i}\right\}$. The map $\rho: s_{i} \mapsto s_{i+1}$ induces an automorphism $\rho$ on $\tilde{A}_{n}$ which we call the cyclic symmetry.

Each element of a Coxeter group $W$ can be written (in many different ways) as a word with letters in $S$. A word $a$ for an element $w$ is called reduced if the length (number of letters) of $a$ is minimal among words representing $w$. The length of a reduced word for $w$ is called the length $l(w)$ of $w$.

Given $u, w \in W$, say that $u \leq w$ in the Bruhat order if some reduced word for $w$ contains as a subword some reduced word for $u$ (in which case any reduced word for $w$ contains a reduced word for $u$ ). It is immediate that Bruhat order is a finitary poset. The cyclic symmetry of $\tilde{A}_{n}$ is an automorphism of the Bruhat order on $\tilde{A}_{n}$ and the map $x \mapsto x^{-1}$ is an automorphism as well.

The following two propositions follow immediately from the definition of Bruhat order. The latter is the well-known "lifting property."

Proposition 4.1. Suppose $u \leq x, v \leq y, l(x y)=l(x)+l(y)$ and $l(u v)=l(u)+l(v)$. Then $u v \leq x y$.

Proposition 4.2. If $u, w \in W$ and $s \in S$ have $w>w s$ and $u>u s$, then the following are equivalent:

(i) $w \geq u$

(ii) $w \geq u s$

(iii) $w s \geq u s$

An equivalent definition of Bruhat order is as follows: A reflection is any element of $W$ conjugate to some $s \in S$, and the set of reflections is denoted $T$. For any reflection $t$ and any element $u$, if $l(u)<l(u t)$ then $u \leq u t$. Bruhat order is the transitive closure of such relations. The inversion set of $w \in W$ is $I(w):=\{t \in T: l(t w)<l(w)\}$. The weak order on $W$ is the partial order with $u \leq v$ if and only if $I(u) \subseteq I(v)$. If $u \leq v$ in weak order then $u \leq v$ in Bruhat order.

When $J$ is any subset of $S$, the subgroup of $W$ generated by $J$ is another Coxeter group, called the parabolic subgroup $W_{J}$. It is known that for any $w \in W$ and $J, K \subseteq S$, the double coset $W_{J} \cdot w \cdot W_{K}$ has a unique Bruhat minimal element ${ }^{J} w^{K}$, and $w$ can be factored (non-uniquely) as $w_{J} \cdot{ }^{J} w^{K} \cdot w_{K}$, where $w_{J} \in W_{J}$ and $w_{K} \in W_{K}$, such that $l(w)=l\left(w_{J}\right)+l\left({ }^{J} w^{K}\right)+l\left(w_{K}\right)$. We have ${ }^{J} w^{K}=\left({ }^{J} w\right)^{K}={ }^{J}\left(w^{K}\right)$. The subset ${ }^{J} W^{K}$ 
consisting of the minimal coset representatives is called a double or two-sided quotient of $W$.

The more widely used one-sided quotients are obtained by letting $J=\emptyset$ or $K=\emptyset$, in which case we write the quotient as $W^{K}$ or ${ }^{J} W$. In the case of one-sided quotients, the factorization $w=w^{K} \cdot w_{K}$ is unique, and furthermore, if $x \in W^{K}$ and $y \in W_{K}$ then $l(x y)=l(x)+l(y)$. The finite case of the following proposition is [14, Proposition 31].

Proposition 4.3. The quotient ${ }^{J} W^{K}$ is an order quotient of $W$.

Proof. We verify the conditions of Definition 3.1. As mentioned above, condition (a) is known. The proof of condition (b) when $W$ is finite can be found in [14, Proposition 31], and the same proof goes through in general. To verify condition (c), let $x, y \in W$ have ${ }^{J} x^{K} \leq y$ and make a particular choice of $x_{J}, x_{K}, y_{J}$ and $y_{K}$ as follows: Write $x=x_{J} \cdot{ }^{J} x$ so that $x_{J} \in W_{J},{ }^{J} x \in{ }^{J} W$ and $l(x)=l\left(x_{J}\right)+l\left({ }^{J} x\right)$. Write ${ }^{J} x=\left({ }^{J} x\right)^{K}\left({ }^{J} x\right)_{K}$ so that $\left({ }^{J} x\right)^{K} \in W^{K},\left({ }^{J} x\right)_{K} \in W_{K}$ and $l\left({ }^{J} x\right)=l\left(\left({ }^{J} x\right)^{K}\right)+l\left(\left({ }^{J} x\right)_{K}\right)$. We have $\left({ }^{J} x\right)^{K}={ }^{J} x^{K}$, so we write $x=x_{J} \cdot{ }^{J} x=x_{J} \cdot{ }^{J} x^{K} \cdot x_{K}$. Using the same process we write $y=y_{J} \cdot{ }^{J} y=y_{J} \cdot{ }^{J}{ }^{K} \cdot y_{K}$.

Bruhat order is directed, so choose $z_{K}$ to be some upper bound for $x_{K}$ and $y_{K}$ in $W_{K}$. Let $z:={ }^{J} y^{K} \cdot z_{K}$. Because ${ }^{J} y^{K} \in{ }^{J} W^{K} \subset W^{K}$ and $z_{K} \in W_{K}$, we have $l(z)=l\left({ }^{J} y^{K}\right)+l\left(z_{K}\right)$, so by Proposition 4.1, $z \geq{ }^{J} x^{K} \cdot x_{K}={ }^{J} x$ and $z \geq{ }^{J} y^{K} \cdot y_{K}={ }^{J} y$. Write $z=z_{J} \cdot{ }^{J} z$ so that ${ }^{J_{z}} \in{ }^{J} W, z_{J} \in W_{J}$ and $l(z)=l\left(z_{J}\right)+l\left({ }^{J} z\right)$. By condition (b), ${ }^{J} z \geq{ }^{J} x$ and ${ }^{J} z \geq{ }^{J} y$. Choose $v_{J}$ to be some upper bound for $x_{J}$ and $y_{J}$ in $W_{J}$ and let $v:=v_{J} \cdot{ }^{J} z$. As before, by Proposition 4.1, $v \geq x_{J} \cdot{ }^{J} x=x$ and $v \geq y_{J} \cdot{ }^{J} y=y$. It remains to show that ${ }^{J} v^{K}={ }^{J} y^{K}$. Since $v=v_{J} \cdot{ }^{J_{z}}=v_{J}\left(z_{J}\right)^{-1} z=v_{J}\left(z_{J}\right)^{-1}\left({ }^{J} y^{K}\right) z_{K}$, we have $v \in W_{J} \cdot{ }^{J} y^{K} \cdot W_{K}$, so by uniqueness of minimal coset representatives, ${ }^{J} v^{K}={ }^{J} y^{K}$.

Projections onto one- or two-sided quotients characterize Bruhat order in a sense made precise by the following theorem due to Deodhar [6], in which $\langle s\rangle:=S-\{s\}$ for each $s \in S$.

Theorem 4.4. Let $(W, S)$ be a Coxeter system and let $v, w \in W$. Then

(i) $v \leq w$ if and only if for every $s \in S$ we have ${ }^{\langle s\rangle} v \leq\langle s\rangle$.

(ii) $v \leq w$ if and only if for every $s \in S$ we have $v^{\langle s\rangle} \leq w^{\langle s\rangle}$.

(iii) $v \leq w$ if and only if for every $s, t \in S$ we have $\langle s\rangle^{\langle t\rangle} \leq\langle s\rangle w^{\langle t\rangle}$.

An element $x \neq 1$ of $W$ is called bigrassmannian if it is contained in ${ }^{\langle s\rangle} W^{\langle t\rangle}$ for some (necessarily unique) $s, t \in S$. Equivalently, $x$ is bigrassmannian if there is a unique $s \in S$ such that $s x<x$ and a unique $t \in S$ such that $x t<x$. The following result was proven in [11, Théoréme 3.6] for finite $W$. The result for general $W$ is an immediate corollary of Theorem 4.4(iii).

Corollary 4.5. A join-irreducible in the Bruhat order on $W$ is bigrassmannian.

Proof. Let $w \in W$. If $u \geq\langle s\rangle w^{\langle t\rangle}$ for every $s$ and $t$ then ${ }^{\langle s\rangle} u^{\langle t\rangle} \geq\langle s\rangle w^{\langle t\rangle}$ so $u \geq w$. Thus $w$ is the join of the set $\left\{{ }^{\langle s\rangle} w^{\langle t\rangle}: s, t \in S\right\}$. If $w$ is not bigrassmannian it is not contained in this set and thus is not join-irreducible. 
Corollary 4.5 and Proposition 3.3 immediately imply the following proposition. Assertion (i) is due to Geck and Kim [9, Corollary 2.8] in the finite case.

Proposition 4.6. For a Coxeter group $W$ under the Bruhat order:

(i) $\operatorname{Irr}(W)=\cup_{s, t \in S} \operatorname{Irr}\left({ }^{\langle s\rangle} W^{\langle t\rangle}\right)$ and

(ii) $\operatorname{Dis}(W)=\cup_{s, t \in S} \operatorname{Dis}\left({ }^{\langle s\rangle} W^{\langle t\rangle}\right)$.

The following fact is useful in finding dissectors in Bruhat order on infinite Coxeter groups. Note the use of both square brackets and round brackets in the statement.

Lemma 4.7. If $x \in W^{\langle s\rangle}$ and $x \neq 1$, then

$$
W-U[x]=\bigcup_{y \in W-U(x s)} y W_{\langle s\rangle}
$$

Proof. Suppose for the sake of contradiction that there exists an element $z$ of the right hand side with $z \geq x$, and choose $z$ to be of minimal length among such elements. Thus $z$ is in one of the cosets on the right hand side, so let $y$ be the minimal coset representative, and write $z=y w$ for some $w \in W_{\langle s\rangle}$. If $w=1$ then $y=z$, so $y \geq x$, contradicting the fact that $y \ngtr x s$. If $w \neq 1$ then choose $t \in S$ such that $w t<w$. Since $w \in W_{\langle s\rangle}$, we have $t \neq s$, so $w t \in W_{\langle s\rangle}$ and thus $z>z t$. Since $x \in W^{\langle s\rangle}$, we have $x t>x$, so by Proposition $4.2 z t \geq x$. Since $z t \in y W_{\langle s\rangle}$, this is a contradiction of our choice of $z$ to be of minimal length among elements of the right hand side which are $\geq x$.

Conversely, suppose $z$ is not an element of the right hand side. In other words, writing $z=z^{\langle s\rangle} \cdot z_{\langle s\rangle}$ as in Proposition 4.3, we have $z^{\langle s\rangle}>x s$. Since $x>x s$ and $z^{\langle s\rangle}>z^{\langle s\rangle} s$, by Proposition $4.2 z^{\langle s\rangle} \geq x$, and therefore $z \geq x$, or in other words, $z$ is not an element of the left hand side.

Proposition 4.8. For a Coxeter group $W$, the following are equivalent:

(i) $W_{J}$ is finite for any $J \subsetneq S$.

(ii) For any $x \in W$ the set $W-U[x]$ is finite.

Proof. For any $J \subsetneq S$ and $s \in(S-J)$, we have $W_{J} \subseteq W-U[s]$, and therefore (ii) implies (i). Conversely, suppose $W_{J}$ is finite for all $J \subsetneq S$, let $x \in W$ and proceed by induction on $l(x)$. The case $l(x)=0$ is trivial so suppose $l(x) \geq 1$. If $x$ is not join-irreducible, then $x=\vee D(x)$, so $U[x]=\bigcap_{a \in D(x)} U[a]$. Thus $W-U[x]=\bigcup_{a \in D(x)}(W-U[a])$ and each term in this finite union is finite by induction. If $x$ is join-irreducible, then in particular by Proposition 4.6, $x \in W^{\langle s\rangle}$ for some $s$. Now Lemma 4.7 writes $W-U[x]$ as a union of sets each of which is finite. By induction, the union is over a finite number of terms.

The affine Coxeter groups and the compact hyperbolic Coxeter groups satisfy the conditions of Proposition 4.8 (see [10] for definitions). If $W$ satisfies the conditions of Proposition 4.8 then $x \in W$ is a dissector if and only if it is a strong dissector. In particular, to apply Theorem 1.2 to $W=\tilde{A}_{n}$ we need only look for strong dissectors. 


\section{Coxeter Groups of Infinite Order Dimension}

In this section we exhibit a large class of Coxeter groups for which the Bruhat order has infinite dimension. To do this we appeal to Theorem 1.2 and to Proposition 5.1, below. A nontrivial element $x \in W$ is called rigid if it admits exactly one reduced word.

Proposition 5.1. If $x$ is rigid then it is a dissector.

Proof. The proof is by induction on $l(x)$. If $l(x)=1$, then $x=s$ for some $s \in S$ and $W-U[x]=W_{\langle s\rangle}$, which is directed by Proposition 4.3. If $l(x)>1$, then let $s$ be the unique element of $S$ such that $x s<x$. Then $x s$ is rigid, so by induction $W-U[x s]$ is directed. By Lemma 4.7, $W-U[x]=\bigcup_{y \in W-U(x s)} y W_{\langle s\rangle}$. Let $u$ and $v$ be elements of $\bigcup_{y \in W-U(x s)} y W_{\langle s\rangle}$. Specifically, $u=u^{\langle s\rangle} \cdot u_{\langle s\rangle}$ and $v=v^{\langle s\rangle} \cdot v_{\langle s\rangle}$ with $u^{\langle s\rangle}, v^{\langle s\rangle} \in W-U(x s)$. Since $(x s) s=x>x s$, the element $x s$ cannot be in $W^{\langle s\rangle}$ unless $x s=1$, but the latter is ruled out because $l(x)>1$. Thus $u^{\langle s\rangle}, v^{\langle s\rangle} \in W-U[x s]$. Since $W-U[x s]$ is directed, there is an element $w \in W-U[x s]$ with $w \geq u^{\langle s\rangle}$ and $w \geq v^{\langle s\rangle}$. So also $w^{\langle s\rangle} \geq u^{\langle s\rangle}$ and $w^{\langle s\rangle} \geq v^{\langle s\rangle}$. Since $W_{\langle s\rangle}$ is directed, there is an element $z \in W_{\langle s\rangle}$ with $z \geq u_{\langle s\rangle}$ and $z \geq v_{\langle s\rangle}$. Thus by Proposition 4.1, $w^{\langle s\rangle} z$ is an upper bound for $u$ and $v$ in $\bigcup_{y \in W-U(x s)} y W_{\langle s\rangle}$.

As an example of the application of Proposition 5.1, consider the universal or free Coxeter group $U_{n}$ with generators $S=\left\{s_{1}, s_{2}, \ldots s_{n}\right\}$ and $m(s, t)=\infty$ for each $s, t \in S$. Every non-trivial element of $U_{n}$ is rigid, so $\operatorname{Dis}\left(U_{n}\right)=U_{n}-\{1\}$, and the order dimension of $U_{n}$ is equal to its width, which is infinite for $n \geq 3$. More generally, if a Coxeter group $W$ has arbitrarily many rigid elements of the same length, then these collections of elements form antichains of dissectors, so $W$ has infinite order dimension.

Rigid elements are in particular paths in the Coxeter graph $\Gamma$. Specifically, a rigid path in $\Gamma$ is a nonempty sequence of vertices of $\Gamma$ such that each consecutive pair in the sequence is an edge in $\Gamma$ and such that the path never traverses an edge of weight $m$ more than $m-2$ times in a row. Rigid elements in $W$ are exactly rigid paths in $\Gamma$. Given two rigid paths $a$ and $b$ in $\Gamma$, say $a$ precedes $b$ if $a b$ is rigid. If $a$ precedes $b, b$ precedes $c$ and $b$ contains more than two distinct letters then $a b c$ is rigid.

As pointed out in [17], an irreducible Coxeter group $W$ with Coxeter graph $\Gamma$ has only finitely many rigid elements if and only if $\Gamma$ is acyclic, has no edges of infinite weight, and has at most one edge of weight greater than or equal to 4. To keep the number of rigid elements of the same length bounded, each of these conditions can be relaxed only very slightly.

Proposition 5.2. Let $W$ be an irreducible Coxeter group with Coxeter graph $\Gamma$. The group $W$ has arbitrarily many rigid elements of the same length if and only if at least one of the following conditions hold:

1. The graph $\Gamma$ contains at least two cycles.

2. The graph $\Gamma$ contains both an edge of weight at least 4 and a cycle. 
3. The graph $\Gamma$ contains an edge of weight at least 4 and another edge of weight at least 6 .

\section{The graph $\Gamma$ contains at least 3 edges of weight at least 4.}

Proof. We give only a sketch, leaving out some straightforward details.

An induced subgraph of a Coxeter graph will be called a core if it consists of a single edge with label infinity, a single cycle, or a path beginning with an edge of weight at least 4 and ending with a different edge of weight at least 4 , with all other edges unlabeled.

Suppose that $W$ has infinitely many rigid elements but satisfies none of the conditions of Proposition 5.2. Then in particular, $\Gamma$ contains a unique core. Furthermore, if the core is a cycle then it is simply laced and if it is a path then it begins and ends with edges labeled 4 or 5 . The rest of $\Gamma$ consists of disjoint branches: simply laced acyclic induced subgraphs each connected to the core by a single edge. Rigid paths cannot turn around within branches, so each rigid path in $\Gamma$ consists of a rigid path in a branch followed by a rigid path in the core, followed by another rigid path in a branch. Any of these three components of the path might be empty. There are only finitely many rigid paths contained in branches, and it is straightforward to give a uniform bound (independent of length) on the number of rigid paths of a given length contained in the core. Thus there is a uniform bound on the number of rigid paths in $\Gamma$ of a given length.

Now suppose $\Gamma$ meets at least one of the conditions of Proposition 5.2. In particular, $\Gamma$ contains some core $C$ with more than two vertices. If $\Gamma$ has at least one cycle, we take $C$ to be one of the cycles. One easily finds a rigid path $a$ in $C$ such that $a$ precedes itself. Specifically, if $C$ is a cycle, let $a$ be a path around the cycle visiting each vertex exactly once. If $C$ is a path, let $a$ begin at one end of the path, traverse the path to the other end and return, stopping one vertex before the starting point. We call $a$ a refrain in $C$.

Given a refrain $a$, any rigid path $b \neq a$ with more than two distinct letters which both precedes $a$ and is preceded by $a$ is called a verse for $a$. Using a refrain $a$ and a verse $b$ one constructs, for each $0 \leq j<k$, a rigid path $a^{j} b a^{k-j-1}$. For each fixed $k$, these are $k$ distinct rigid words of the same length. Thus the proof can be completed by constructing a verse for $a$.

The conditions of Proposition 5.2 guarantee that one or more of the following cases occurs:

(i) there is an edge of weight at least 4 not contained in $C$;

(ii) $C$ is a path one of whose terminal edges has weight at least 6 ;

(iii) $C$ is a cycle and $\Gamma$ contains another cycle; or

(iv) $C$ is a cycle one of whose edges is weighted at least 4 .

In each of these cases, it is straightforward to construct a verse for $a$.

For any two partially ordered sets $P$ and $Q$, we can see that

$$
\max \{\operatorname{dim}(P), \operatorname{dim}(Q)\} \leq \operatorname{dim}(P \times Q) \leq \operatorname{dim}(P)+\operatorname{dim}(Q) .
$$


It follows that a finitely generated Coxeter group has infinite order dimension if and only if at least one of its irreducible components does. By Propositions 5.1 and 5.2, we can form several large classes of Coxeter groups of infinite order dimension. On the other hand, Theorem 1.1 establishes an infinite class of infinite Coxeter groups of finite order dimension, so the following question seems appropriate:

Question 5.3. For which Coxeter groups does the Bruhat order have finite order dimension, and what are these dimensions?

\section{Affine Permutations}

In this section we review a combinatorial description, due to Lusztig [12], of the affine Coxeter group $\tilde{A}_{n-1}$, and a criterion due to Björner and Brenti [2], for making Bruhat comparisons. We rewrite the criterion in terms of infinite tableaux. A similar criterion was given by H. Eriksson in [7]. In this section and the next it is more convenient to work with $\tilde{A}_{n-1}$. Subscripts labeling the generators should be interpreted $\bmod n$.

Let $\tilde{S}_{n}$ be the set of affine permutations, that is, permutations $x$ of $\mathbb{Z}$ with the following properties:

$$
x(i+n)=x(i)+n,
$$

for all $i \in \mathbb{Z}$, and

$$
\sum_{i=1}^{n} x(i)=\left(\begin{array}{c}
n+1 \\
2
\end{array}\right) .
$$

An affine permutation $x$ is uniquely identified by the values $x(1), x(2), \ldots, x(n)$, called the window of $x$. Affine permutation are specified by writing the window values in square brackets, separated by commas. The set $\tilde{S}_{n}$ forms a group under composition, and is generated by $S=\left\{s_{1}, s_{2}, \ldots, s_{n}\right\}$, with

$$
s_{j}=[1,2, \ldots, j-1, j+1, j, j+2, \ldots, n]
$$

for $j \in[n-1]$ and

$$
s_{n}=[0,2,3, \ldots, n-1, n+1] .
$$

Putting $s_{n+1}=s_{1}$, we have $m\left(s_{j}, s_{j+1}\right)=3$ for all $j \in[n]$, and all the other pairwise orders are 2 . There are no other relations in the affine permutation group $\tilde{S}_{n}$, so $\tilde{S}_{n}$ is isomorphic to the Coxeter group $\tilde{A}_{n-1}$.

The length of $x \in \widetilde{S}_{n}$ is

$$
l(x)=\#\{(i, j) \in[n] \times \mathbb{Z}: i<j, x(i)>x(j)\} .
$$

The reflections in $\tilde{S}_{n}$ are infinite products of transpositions

$$
t_{i, j}:=\prod_{r \in \mathbb{Z}}(i+r n, j+r n)
$$


for $i, j \in \mathbb{Z}$ and $i \not \equiv j \bmod n$. Thus if $t_{i, j}$ is a reflection with $i<j$ and $x \in \tilde{S}_{n}$ has $x(i)<x(j)$, then $x \leq x t_{i, j}$ in Bruhat order. All other Bruhat relations are obtained by transitivity.

Björner and Brenti [2, Theorem 6.5] gave a criterion for making Bruhat comparisons on $\tilde{S}_{n}$, similar to the Tableau Criterion on certain finite Coxeter groups. For $x \in \tilde{S}_{n}$ and $i, j \in \mathbb{Z}$, define

$$
x[i, j]:=\#\{k \in \mathbb{Z}: k \leq i, x(k) \geq j\} .
$$

Then $u \leq v$ in Bruhat order if and only if $u[i, j] \leq v[i, j]$ for all $i, j \in \mathbb{Z}$. Björner and Brenti also show that it is enough to check $i \in[n]$ and that for each $u$ and $v$, there is only a finite number of values $j$ which must be checked. ${ }^{1}$ To make this criterion resemble more closely the tableau criterion on the symmetric group, we define an infinite tableau $T_{a, b}(u)$ as follows. For each $a, b \in \mathbb{Z}$ with $b \leq a$, let $T_{a, b}(u)$ be the entry at position $b$ in the increasing rearrangement of the set $\{u(i): i \in \mathbb{Z}, i \leq a\}$. That is, rearrange the set in increasing order and place the rearranged values so that they occupy the integer positions of $(-\infty, a]$. The easy proof of the following proposition is omitted.

Proposition 6.1. Let $u, v \in \tilde{S}_{n}$. Then $u[i, j] \leq v[i, j]$ for all $i, j \in \mathbb{Z}$ if and only if $T_{a, b}(u) \leq T_{a, b}(v)$ for all $a, b \in \mathbb{Z}$ with $b \leq a$.

We now make note of some properties of the infinite tableau $T_{a, b}(u)$. Properties (i) to (iv) follow immediately from the definitions of $\tilde{S}_{n}$ and $T_{a, b}(u)$. Property (v) follows from the fact that the identity permutation is minimal in $\tilde{S}_{n}$. We give proofs of Properties (vi) and (vii).

Proposition 6.2. Let $u \in \tilde{S}_{n}, a, b \in \mathbb{Z}$ and $b \in(-\infty, a]$ and write $T_{a, b}$ for $T_{a, b}(u)$. Then

(i) $T_{a, b-1}<T_{a, b}$.

(ii) $T_{a+1, b} \leq T_{a, b} \leq T_{a+1, b+1}$.

(iii) $T_{a+n, b+n}=T_{a, b}+n$.

(iv) If $j$ occurs as an entry in row a of $T_{a, b}$ then $j-n$ also occurs in row a.

(v) $T_{a, b}(x) \geq b$.

(vi) If $T_{a, b}=T_{a, b-n}+n$ then $T_{a, b}=b$.

(vii) For each fixed a there is a $B$ such that $T_{a, b}=b$ for every $b \leq B$.

\footnotetext{
${ }^{1}$ Although [2, Theorem 6.5] looks different from what we quote here, one verifies that the quantity $\varphi_{\{x(j), x(j+1), \ldots, x(j+n-1)\}}(i+1)$ in the statement of $[2$, Theorem 6.5$]$ is equal to $\left(x^{-1}\right)[i, j]$. Since the map $x \mapsto x^{-1}$ is an automorphism of Bruhat order, the two criteria are equivalent. The formulation given above was communicated to the authors in 2001 by Björner and Brenti and will appear in [3].
} 
Proof. To prove Property (vi), suppose $T_{a, b}=T_{a, b-n}+n$. Then by (iii), $T_{a-n, b-n}=T_{a, b-n}$. Therefore elements in $\{u(i): i \leq a\}-\{u(i): i \leq a-n\}$ all occur to the right of column $b-n$ in rows $a-n$ through $a$ of $T$. Thus

$$
\begin{aligned}
\sum_{i=a-n+1}^{a} u(i) & =\sum_{j=b-n+1}^{a} T_{a, j}-\sum_{j=b-n+1}^{a-n} T_{a-n, j} \\
& =\sum_{j=b-n+1}^{a} T_{a, j}-\sum_{j=b-n+1}^{a-n}\left(T_{a, j+n}-n\right) \\
& =\sum_{j=b-n+1}^{a} T_{a, j}-\sum_{j=b+1}^{a}\left(T_{a, j}-n\right) \\
& =n(a-b)+\sum_{j=b-n+1}^{b} T_{a, j}
\end{aligned}
$$

Row $a$ strictly increases from $T_{a, b-n}$ to $T_{a, b}=T_{a, b-n}+n$, so this sequence of values is $T_{a, b-n}, T_{a, b-n}+1, \ldots, T_{a, b-n}+n$. Therefore

$$
\sum_{j=b-n+1}^{b} T_{a, j}=n \cdot T_{a, b-n}+\left(\begin{array}{c}
n+1 \\
2
\end{array}\right) .
$$

On the other hand, combining Equations (1) and (2), we obtain

$$
\sum_{i=a-n+1}^{a} u(i)=n(a-n)+\left(\begin{array}{c}
n+1 \\
2
\end{array}\right) .
$$

Equating the two expressions for $\sum_{i=a-n+1}^{a} u(i)$ and solving yields $T_{a, b-n}=b-n$, so $T_{a, b}=b$.

Properties (i) and (vi) imply that if $T_{a, b} \neq b$ then $T_{a, b-n}<T_{a, b}-n$. In light of (v), this implies (vii).

A function $T:\{a, b \in \mathbb{Z}: b \leq a\} \rightarrow \mathbb{Z}$ satisfying the conditions of Proposition 6.2 will be called an affine monotone triangle. Affine monotone triangles $T_{a, b}$ are represented as arrays of $n$ rows corresponding to $a \in[n]$, with a vertical line at the left of the array to indicate that all entries to the left of the line have $T_{a, b}=b$. Entries with $T_{a, b}=b$ are called trivial. So, for example, when $n=3$, the permutation $[3,-2,5]$ has

$$
T([3,-2,5])=\mid \begin{array}{rrrrrrr}
-3 & -1 & 0 & 2 & 3 & & \\
-3 & -2 & -1 & 0 & 2 & 3 & \\
-3 & -2 & -1 & 0 & 2 & 3 & 5
\end{array} .
$$




\section{Dissectors in $\tilde{A}_{n-1}$}

In this section we prove the lower bound of Theorem 1.1, by exhibiting an antichain of dissectors. Two descriptions of the dissectors are useful. The first description helps in the proof that they indeed are dissectors, while the other description is useful in determining the order relations among them.

For $a \in[n]$, and $b, c \in \mathbb{Z}$ with $b \leq a$ and $b \leq c$, define $J_{a, b, c}$ to be the unique Bruhat minimal element in the set $\left\{x \in \tilde{S}_{n}: T_{a, b}(x) \geq c\right\}$, if such an element exists. If $b=c$ then $J_{a, b, c}$ is the identity. There are choices of $a, b$ and $c$ for which $J_{a, b, c}$ is undefined. For example, in $\tilde{S}_{3}$, the infinite tableaux for the affine permutations $[3,-2,5]$ and $[5,0,1]$ are both minimal among affine monotone triangles $T$ with $T_{1,0} \geq 2$. Similarly, define $M_{a, b, c}$ to be the unique Bruhat maximal element in the set $\left\{x \in \tilde{S}_{n}: T_{a, b}(x)<c\right\}$, if such an element exists. If $J_{a, b, c}$ and $M_{a, b, c}$ are both defined and represent affine permutations for a triple $(a, b, c)$, then $J_{a, b, c}$ is a dissector of $\tilde{S}_{n}$ with $\beta\left(J_{a, b, c}\right)=M_{a, b, c}$. A similar approach to finding dissectors in certain finite Coxeter groups was taken in [11] and [14].

The second description of dissectors is as left-justified rectangles in the array:

$\begin{array}{ccccccc}s_{1} & s_{0} & s_{-1} & \cdot & \cdot & \cdot & s_{-n+3} \\ s_{2} & s_{1} & s_{0} & \cdot & \cdot & \cdot & s_{-n+4} \\ s_{3} & s_{2} & s_{1} & \cdot & \cdot & \cdot & s_{-n+5} \\ \cdot & \cdot & \cdot & & & \cdot \\ \cdot & \cdot & \cdot & & & \cdot \\ \cdot & \cdot & \cdot & & & & \cdot\end{array}$.

This array has infinitely many rows of length $n-1$, where the $i$ in $s_{i}$ is to be interpreted $\bmod n$. Rectangles are interpreted as elements of $A_{n-1}$ by reading the characters in the usual direction for reading written English. So for example, the rectangle

$\begin{array}{cccc}s_{1} & s_{0} & s_{-1} & s_{-2} \\ s_{2} & s_{1} & s_{0} & s_{-1} \\ s_{3} & s_{2} & s_{1} & s_{0}\end{array}$

stands for the word $s_{1} s_{n} s_{n-1} s_{n-2} s_{2} s_{1} s_{n} s_{n-1} s_{3} s_{2} s_{1} s_{n}$. The rectangle which is $i$ columns wide and $k$ rows long, and whose top left corner is $s_{j}$ is referred to as $R_{i, j, k}$. The remainder of the section is devoted to proving the following lemmas:

Lemma 7.1. If $i+k \leq n$ then $J_{j+k-i, j-i+1, j+1}$ is defined and represents the affine permutation $R_{i, j, k}$.

Lemma 7.2. $M_{a, b, c}$ is defined and represents an affine permutation whenever $b \leq a$ and $b<c$.

Lemma 7.3. The set $\left\{R_{i, j, k}: i+k=n\right\}$ is an antichain in Bruhat order.

Lemmas 7.1 and 7.2 imply that the rectangles $R_{i, j, k}$ are dissectors. Lemma 7.3 exhibits an antichain in $\operatorname{Dis}\left(\tilde{A}_{n-1}\right)$ with $n(n-1)$ elements, so that by Theorem 1.2 , the order 
dimension of the Bruhat order on $\tilde{A}_{n-1}$ is at least $n(n-1)$. This is the lower bound in Theorem 1.1.

When $i=1$ the element $R_{i, j, k}$ is rigid for any $j$ and $k$, and therefore is a dissector by Proposition 5.1. These rectangles are called cyclic words because they correspond to cyclic paths in the Coxeter graph for $\tilde{A}_{n-1}$. There are also cyclic words in the opposite direction. Computer investigations suggest that the cyclic words and the rectangles with $i+k \leq n$ are the only dissectors in $\tilde{S}_{n}$

Proof of Lemma \%.1. Because of the cyclic symmetry of $\tilde{A}_{n-1}$, for each fixed $i$ and $k$, checking Lemma 7.1 for one particular $j$ is enough. Specifically, the map $\rho$ which sends $s_{i}$ to $s_{i+1}$ for each $i \in[n]$ corresponds to moving the window one position to the left and then adding one to each entry in the window. The corresponding map on tableaux is $\rho(T)_{a, b}=T_{a-1, b-1}+1$. Thus if $J_{a, b, c}$ is defined, $\rho\left(J_{a, b, c}\right)=J_{a+1, b+1, c+1}$. On the other hand $\rho\left(R_{i, j, k}\right)=R_{i, j+1, k}$.

For convenience, we consider the case when $j=i-k+n$. In effect this fixes the bottom-right element of the rectangle to be $s_{n}$. By induction on $k$, it can be verified that for $i+k \leq n$, the rectangle $R_{i, i-k+n, k}$ is the affine permutation whose window is

$$
[1-k, \ldots, i-k, i+1, \ldots, n-k, n-k+i+1, \ldots, n+i]
$$

That is,

$$
R_{i, i-k+n, k}(m)=\left\{\begin{array}{ll}
m-k & \text { if } 1 \leq m \leq i \\
m & \text { if } i+1 \leq m \leq n-k \\
m+i & \text { if } n-k+1 \leq m \leq n
\end{array} .\right.
$$

So, for example for $n=7$,

$$
R_{2,6,3}=[-2,-1,3,4,7,8,9] .
$$

We now show that $T\left(R_{i, i-k+n, k}\right)$ is minimal under componentwise comparison among all affine monotone triangles whose $(n, n-k+1)$ entry is at least $n+i-k+1$. It is awkward to represent $T\left(R_{i, i-k+n, k}\right)$ in its full generality, so we continue our example to illustrate the argument. When $n=7$,

$$
T\left(R_{2,6,3}\right)=\mid \begin{array}{rrrrrrrrrrrr}
-2 & 0 & 1 & 2 & & & & & & \\
-2 & -1 & 0 & 1 & 2 & & & & & \\
-2 & -1 & 0 & 1 & 2 & 3 & & & & \\
-2 & -1 & 0 & 1 & 2 & 3 & 4 & & & \\
-2 & -1 & 0 & 1 & 2 & 3 & 4 & 7 & & \\
-2 & -1 & 0 & 1 & 2 & 3 & 4 & 7 & 8 & \\
-2 & -1 & 0 & 1 & 2 & 3 & 4 & 7 & 8 & 9
\end{array}
$$

For $a \in[i, n]$ and $b \leq n-k, T_{a, b}$ achieves the lower bound of property (v). Since $i+k \leq n$, we have $i \leq n-k$, so in particular, every entry in row $i$ achieves this lower bound. The lower bound of $(\mathrm{v})$ is also achieved for $a \in[1, i-1]$ and $b \leq a-k$. For $a \in[i, n]$ with $b \geq n-k+1$, the entries are increasing by ones as one moves to the right and are constant 
in columns. Thus by (i) and (ii) they are as small as possible subject to the constraint that $T_{n, n-k+1} \geq n+i-k+1$.

By property (iii), for $b \in[-k+1,0]$ we have $T_{0, b}=T_{n, n+b}+n=b+i$. Since the entries in row $n$ are as small as possible, by property (ii) for $r \in[1, i]$ and $b \in[-k+1,0]$, the smallest possible entry $T_{r, b+r}$ is $b+i$. The corresponding entries of $T\left(R_{i, i-k+n, k}\right)$ achieve this bound.

Proof of Lemma 7.2. By cyclic symmetry, we need only consider the case where $a=n$. We claim that $M_{n, b, c}$ represents the affine permutation $x_{n, b, c}$ whose window is

$$
\left[n^{2}-n b+c-1, c-2, \ldots, c-n+1, c+b n-c n\right] .
$$

That is,

$$
M_{n, b, c}(m)= \begin{cases}n^{2}-n b+c-1 & \text { if } m=1 \\ c-m & \text { if } 2 \leq m \leq n-1 \\ c+b n-c n & \text { if } m=n\end{cases}
$$

So, for $n=7$,

$$
x_{7,5,7}=[20,5,4,3,2,1,-7]
$$

and $T\left(x_{7,5,7}\right)$ is

$$
\begin{array}{|rrrrrrrrrrrrrrrr}
-6 & -5 & -4 & -3 & -2 & -1 & 6 & 13 & 20 & & & & & & \\
-6 & -5 & -4 & -3 & -2 & -1 & 5 & 6 & 13 & 20 & & & & & \\
-6 & -5 & -4 & -3 & -2 & -1 & 4 & 5 & 6 & 13 & 20 & & & & \\
-6 & -5 & -4 & -3 & -2 & -1 & 3 & 4 & 5 & 6 & 13 & 20 & & & \\
-6 & -5 & -4 & -3 & -2 & -1 & 2 & 3 & 4 & 5 & 6 & 13 & 20 & & \\
-6 & -5 & -4 & -3 & -2 & -1 & 1 & 2 & 3 & 4 & 5 & 6 & 13 & 20 & \\
-7 & -6 & -5 & -4 & -3 & -2 & -1 & 1 & 2 & 3 & 4 & 5 & 6 & 13 & 20
\end{array}
$$

We now show that the affine monotone triangle $T\left(x_{n, b, c}\right)$ is maximal among affine monotone triangles whose $(n, b)$ entry is at most $c-1$. Let $L:=c+b n-c n+1$, so that in the example, $L=-6$. Then row $n$ of $T\left(x_{n, b, c}\right)$ is

$$
\begin{aligned}
\mid L-1, L, \ldots, L+n-2, L+n, \ldots, L+2 n-2, \cdots, c-n+1, \ldots, c-1 \\
n+c-1,2 n+c-1, \ldots, n^{2}-n b+c-1 .
\end{aligned}
$$

In words, the row has trivial entries in (and to the left of) position $L+n-2$. Starting in position $L+n-1$ there are sequences $L+n, \ldots, L+2 n-2, L+2 n, \ldots, L+3 n-2$, etc. up to the sequence $c-n+1, \ldots, c-1$. The row ends with a sequence of entries differing by $n$.

The entries to the right of position $(n, b)$ are as large as possible by Property (iv). The entries in positions $(n, b-n+2)$ to $(n, b-1)$ are as large as possible by Property (i). Now suppose that some affine monotone triangle $T$ agrees with $T\left(x_{n, b, c}\right)$ in positions $(n, b-n+2)$ through $(n, n)$. If $b=c-1$, then we have $T_{n, b}=b$, so all entries to the left in row $n$ are trivial. Otherwise Property (vi) says that $T_{n, b-n} \neq T_{n, b}-n$, and Property 
(iv) says that the value $T_{n, b}-n$ must occur somewhere in the row. Thus we cannot have $T_{n, b-n+1}=T_{n, b}-n+1$, or in other words, we must have $T_{n, b-n+1} \leq T_{n, b}-n \leq c-n-1$. So the entry in position $(n, b-n+1)$ of $T\left(x_{a, b, c}\right)$ is as large as possible. We continue moving left in the row, using Properties (i), (iv) and (vi) in the same manner to show that all the entries in this row are as large as possible until eventually, by Property (vii), the remaining entries in the row are trivial.

Each nontrivial entry in row $n-1$ is equal to the entry one column to the right in row $n$, and thus by property (ii) these entries are as large as possible. By property (iii) the entries in row 0 are also as large as possible. For each $r \in[1, n-1]$, row $r$ is obtained from row $r-1$ by adjoining an element greater than $c-n$. Since the entry at $(0, b-n)$ is $c-n-1$, an entry in row $r$ weakly left of column $b-n$ agrees with the corresponding entry in row 0 and thus by property (ii) is as large as possible.

Each entry in rows 1 through $n-1$ to the right of column $b-n$ is equal to the entry below it and to the right. By property (ii) these entries are as large as possible.

To prove Lemma 7.3 , we observe that $R_{i, j, k}$ is a fully commutative element. A fully commutative element [17] is an element $w$ such that any two reduced words for $w$ are related by commutation of generators. In $R_{i, j, k}$, between any two occurrences of a generator $s$, there occur two distinct generators $t$ and $t^{\prime}$ with $m(s, t)=m\left(s, t^{\prime}\right)=3$. This is enough to ensure that the rectangles $R_{i, j, k}$ are reduced words for fully commutative elements [1]. The following proposition is immediate from the definition of full commutativity.

Proposition 7.4. Let $w$ be a fully commutative element of $W$, let $s_{1} s_{2} \cdots s_{k}$ be a reduced word for $w$, and let $s_{i_{1}} s_{i_{2}} \cdots s_{i_{j}}$ be a subword such that for every $m \in[j-1]$, the generators $s_{i_{m}}$ and $s_{i_{m+1}}$ do not commute. Then $s_{i_{1}} s_{i_{2}} \cdots s_{i_{j}}$ occurs as a subword of every reduced word for $w$.

A subrectangle of $R_{i, j, k}$ is a rectangle that is obtained by deleting columns from the left and/or right of $R_{i, j, k}$ and/or deleting rows from the top and/or bottom of $R_{i, j, k}$. The following proposition is an affine version of [14, Proposition 38].

Proposition 7.5. If $i^{\prime}+k^{\prime} \leq n$ then $R_{i, j, k} \leq R_{i^{\prime}, j^{\prime}, k^{\prime}}$ if and only if $R_{i, j, k}$ is a subrectangle of $R_{i^{\prime}, j^{\prime}, k^{\prime}}$.

Proof. The "if" direction is immediate from the definition of Bruhat order.

Suppose $R_{i, j, k} \leq R_{i^{\prime}, j^{\prime}, k^{\prime}}$, and let $a$ be the word obtained from $R_{i^{\prime}, j^{\prime}, k^{\prime}}$ by reading across rows as described above. Thus some reduced word for $R_{i, j, k}$ is a subword of $a$. But $R_{i, j, k}$ has a subword

$$
s_{j} s_{j-1} \cdots s_{j-i+2} s_{j-i+1} s_{j-i+2} \cdots s_{j-i+k-1} s_{j-i+k}
$$

which satisfies the hypotheses of Proposition 7.4. Therefore, the subword of $a$ which is a reduced word for $R_{i, j, k}$ must itself contain the same subword. For $a$ to contain the letters $s_{j} s_{j-1} \cdots s_{j-i+2} s_{j-i+1}$ in that order, in particular, it must contain the letter $s_{j-1}$ somewhere after an occurrence of $s_{j}$. Thus, because $a$ comes from a rectangle, there is either an occurrence of $s_{j-1}$ immediately to the right of some occurrence of $s_{j}$, or there is an occurrence of $s_{j-1}$ in the position $x$ columns left and $n-1-x$ rows below some 
occurrence of $s_{j}$, for some $x \in[n-2]$. The latter possibility is excluded by the hypothesis that $i^{\prime}+k^{\prime} \leq n$. Proceeding in this manner, we find that some row in $R_{i^{\prime}, j^{\prime}, k^{\prime}}$ contains $s_{j} s_{j-1} \cdots s_{j-i+2} s_{j-i+1}$. For the letters $s_{j-i+2} s_{j-i+1} s_{j-i+2} \cdots s_{j-i+k-1} s_{j-i+k}$ to occur after that row, in that order, there must be at least $k-1$ more rows.

Proof of Lemma 7.3. In $\left\{R_{i, j, k}: i+k=n\right\}$, subrectangle relations are impossible when the dimensions of the rectangles disagree. Two rectangles of the same dimensions but different top-left entries are also not related by the subrectangle order.

Remark 7.6. By Lemma 7.2, the set of meet-irreducibles is contained in the set

$$
\left\{M_{a, b, c}: a \in[n], b \leq a, b<c\right\}
$$

because any other element $x$ can be written

$$
x=\wedge\left\{M_{a, b, T_{a, b}(x)}: a \in[n], T_{a, b}>b\right\} .
$$

By Property (vii) of signed monotone triangles, this is the meet of a finite set. One can prove a version of Theorem 1.2 which bounds the order dimension of a finitary set below the width of the subposet of meet-irreducibles. Thus one might hope to get an upper bound on $\operatorname{dim}\left(\tilde{A}_{n-1}\right)$ as the width of the set of $M_{a, b, c}$ 's. However, computer tests suggest that this width is not finite.

\section{Join-irreducibles in $\tilde{A}_{n}$}

In this section we review root systems and the standard order on dominant weights, quote several results from [18] and use these results to identify the join-irreducibles in the standard order on dominant weights for $A_{n}$. For more details on root systems and Coxeter groups, see [4, 10]. For more on the poset of dominant weights, see [18].

Given a nonzero vector $\alpha$ in a real Euclidean space $V$, let $H_{\alpha}$ be the hyperplane normal to $\alpha$, and let $r_{\alpha}$ be the Euclidean reflection fixing $H_{\alpha}$. A (finite) root system is a finite collection $\Phi$ of vectors in $V$, satisfying the following properties:

(i) $r_{\alpha} \Phi=\Phi$ for any $\alpha \in \Phi$.

(ii) $\alpha \mathbb{R} \cap \Phi=\{ \pm \alpha\}$ for any $\alpha \in \Phi$.

Each root $\alpha \in \Phi$ has a corresponding co-root $\alpha^{\vee}:=2 \alpha /\langle\alpha, \alpha\rangle$. The set $\Phi^{\vee}:=\left\{\alpha^{\vee}: \alpha \in \Phi\right\}$ is also a root system called the co-root system. A root system $\Phi$ is crystallographic if $\left\langle\alpha, \beta^{\vee}\right\rangle \in \mathbb{Z}$ for any $\alpha, \beta \in \Phi$. From here on, we assume that $\Phi$ is crystallographic.

The group $W$ generated by the reflections $r_{\alpha}$ for $\alpha \in \Phi$ is a finite Coxeter group. The rank of a root system $\Phi$, which we denote by $n$, is the dimension of its linear span. Choose any vector $v \in V$ which is not orthogonal to any root in $\Phi$. The set of positive roots of $\Phi$ is $\Phi^{+}:=\{\alpha \in \Phi:\langle\alpha, v\rangle>0\}$. The set $\Delta$ of simple roots of $\Phi$ is the minimal subset of $\Phi$ with the property that every $\alpha \in \Phi^{+}$is in the nonnegative linear span of $\Delta$. In particular $\Delta$ is a basis for the linear span of $\Phi$. 
As a warning to the reader, we point out that the term "lattice" appears in this section in two completely different senses. This is unavoidable, as both usages of the term are completely standard. Besides denoting a poset with meets and joins, the term lattice also denotes a discrete additive subgroup of a vector space. However, the latter usage of the term only appears in this paper within the phrase "root lattice" or "weight lattice."

The weight lattice associated to $\Phi$ is the set

$$
\Lambda:=\left\{\lambda \in \operatorname{Span}(\Phi):\left\langle\lambda, \alpha^{\vee}\right\rangle \in \mathbb{Z} \text { for all } \alpha \in \Phi\right\} .
$$

The elements of $\Lambda$ are called weights. The fundamental weights $\omega_{1}, \ldots, \omega_{n}$ are elements of the span of $\Phi$ defined by the equations $\left\langle\omega_{i}, \alpha_{j}^{\vee}\right\rangle=\delta_{i j}$. A vector $v \in V$ is dominant if $\left\langle v, \alpha^{\vee}\right\rangle \geq 0$ for every $\alpha \in \Delta$. In particular the subset $\Lambda^{+}$of the weight lattice consisting of dominant weights is equal to the nonnegative integer span of $\left\{\omega_{1}, \ldots, \omega_{n}\right\}$. The standard order on the weight lattice $\Lambda$ is the partial order that sets $\lambda \leq \mu$ if and only if $\mu-\lambda$ is in the nonnegative integer span of $\Delta$. The root poset is the restriction of the standard order to the positive roots. (Roots are in particular weights by the crystallographic assumption).

The standard order on dominant weights is the restriction of the standard order to $\Lambda^{+}$. The poset $\Lambda^{+}$is in general not connected. It has one component for each coset of $\Lambda$ modulo the root lattice $\mathbb{Z} \Phi$. Each component of $\Lambda^{+}$is a lattice, and the cover relations were determined explicitly in [18] for general $W$.

From now on, we restrict to the case where $W$ is the Coxeter group $A_{n}$ and choose a corresponding root system with positive roots $\left\{\epsilon_{a}-\epsilon_{b}: 1 \leq b<a \leq n+1\right\}$. Although this root system is defined in $\mathbb{R}^{n+1}$, its span is the hyperplane consisting of vectors whose entries sum to zero, and thus its rank is $n$. The simple roots are $\alpha_{i}=\epsilon_{i+1}-\epsilon_{i}$. Every positive root is $\alpha_{i j}:=\alpha_{i}+\alpha_{i+1}+\cdots \alpha_{j}$ for some $j \geq i$. In particular, $\alpha_{i i}$ means $\alpha_{i}$. Each root $\alpha$ has $\langle\alpha, \alpha\rangle=2$, so that $\alpha^{\vee}=\alpha$. Thus $\left\langle\alpha_{i}, \alpha_{j}\right\rangle$ is 2 if $i=j$, is -1 if $|i-j|=1$ and is 0 otherwise. The root poset for $A_{n}$ has $\alpha_{i j} \leq \alpha_{r s}$ if and only if $[r, s] \subseteq[i, j]$.

The following is [18, Theorem 2.8], specialized to the case where $W=A_{n}$.

Theorem 8.1. Let $\Lambda^{+}$be the standard order on the dominant weights of the root system corresponding to the Coxeter group $A_{n}$. Suppose $\lambda>\mu$ in $\Lambda^{+}$. Then $\lambda$ covers $\mu$ if and only if $\lambda-\mu=\alpha_{i j}$ for a positive root $\alpha_{i j}$ and one of the following holds:

(i) $i=j$, or

(ii) $i<j$ and $\left\langle\mu, \alpha_{k}^{\vee}\right\rangle=0$ for all $k \in[i, j]$.

Theorem 8.1 allows us to determine the join-irreducibles of $\Lambda^{+}$. The minimal element of each component of $\Lambda^{+}$is join-irreducible in $\Lambda^{+}$, but not join-irreducible in that component of $\Lambda^{+}$. Because each component is a lattice, a non-minimal element is join-irreducible (both in $\Lambda^{+}$and in its own component of $\Lambda^{+}$) if and only if it covers exactly one element. The minimal elements of the components are not relevant to the proof of Theorem 1.1, so we refer to these minimal elements as trivial join-irreducibles.

Lemma 8.2. Let $W=A_{n}$ and $\lambda \in \Lambda^{+}$. Then $\lambda$ is a nontrivial join-irreducible if and only if there is a unique $\alpha$ which is minimal in the root poset among positive roots with the property that $\lambda-\alpha \in \Lambda^{+}$. In this case $\lambda \gg(\lambda-\alpha)$. 
Proof. Suppose that $\lambda$ is a nontrivial join-irreducible and suppose that $\beta$ is minimal in the root poset among positive roots with the property that $\lambda-\beta \in \Lambda^{+}$. By Theorem 8.1, there is some positive root $\alpha$ such that $\lambda$ covers $\lambda-\alpha$ and nothing else. So in particular, $(\lambda-\alpha) \geq(\lambda-\beta)$, which implies that $\beta \geq \alpha$ in the root poset. But since $\beta$ is minimal, $\beta=\alpha$, and thus $\alpha$ is the desired unique minimal element.

Conversely, suppose that $\alpha$ is the unique minimal element in the root poset such that $\lambda-\alpha \in \Lambda^{+}$, and suppose that $\lambda$ covers $\lambda-\beta$. Since $\lambda-\beta \in \Lambda^{+}$, we have $\alpha \leq \beta$ in the root poset, so $(\lambda-\alpha) \geq(\lambda-\beta)$ in $\Lambda^{+}$. But since $\lambda$ covers $\lambda-\beta$, we have $\alpha=\beta$, so $\lambda-\alpha$ is the unique element covered by $\lambda$.

Lemma 8.2 can be used to determine the join-irreducibles explicitly as sums of the fundamental weights $\omega_{i}$. To simplify notation, define $\omega_{0}=\omega_{n+1}=0$.

Proposition 8.3. For $W=A_{n}$, the nontrivial join-irreducible elements of $\Lambda^{+}$are exactly the elements of the following forms:

(a) $\omega_{i}+\omega_{j}$ for $1 \leq i<j \leq n$ or

(b) $\omega_{i}+c \omega_{j}+\omega_{k}$ for $0 \leq i<j<k \leq n+1$ and $c \geq 2$.

Proof. Let $\lambda \in \Lambda^{+}$and write $\lambda=c_{1} \omega_{1}+\cdots c_{n} \omega_{n}$. Then $\left\langle\lambda-\alpha_{i}, \alpha_{i}^{\vee}\right\rangle=c_{i}-2$ and for $j \neq i$ we have $\left\langle\lambda-\alpha_{i}, \alpha_{j}^{\vee}\right\rangle \geq\left\langle\lambda, \alpha_{j}^{\vee}\right\rangle$ which is nonnegative because $\lambda$ is dominant. Thus $\lambda-\alpha_{i} \in \Lambda^{+}$if and only if $c_{i} \geq 2$, and by Theorem 8.1, $\lambda$ covers $\lambda-\alpha_{i}$ in this case.

Suppose now that $\lambda \in \Lambda^{+}$is join-irreducible. Then in particular, there is at most one $i$ with $c_{i} \geq 2$. Furthermore, suppose that $c_{i} \geq 1$ and $c_{j} \geq 1$ for some $i<j$, but $c_{i+1}=c_{i+2}=\cdots=c_{j-1}=0$. Then for $k<i$ or $k>j$ we have $\left\langle\lambda-\alpha_{i j}, \alpha_{k}^{\vee}\right\rangle \geq\left\langle\lambda, \alpha_{k}^{\vee}\right\rangle \geq 0$. For $k=i$ or $k=j$ we have $\left\langle\lambda-\alpha_{i j}, \alpha_{k}^{\vee}\right\rangle=c_{k}-2+1 \geq 0$. For $i<k<j$, we have $\left\langle\lambda-\alpha_{i j}, \alpha_{k}^{\vee}\right\rangle=0+1-2+1=0$. Thus $\lambda-\alpha_{i j} \in \Lambda^{+}$. If $i<j<k$ and $c_{i}, c_{j}$ and $c_{k}$ are all nonzero coefficients, with only zero coefficients between them, then both $\lambda-\alpha_{i j}$ and $\lambda-\alpha_{j k}$ are in $\Lambda^{+}$, so by Lemma 8.2, we have $\lambda-\alpha_{j}$ in $\Lambda^{+}$, so in particular $\lambda$ covers $\lambda-\alpha_{j}$, and so by the previous paragraph $c_{j} \geq 2$. Since no other entry is $\geq 2$, these must be the only nonzero entries. Thus $\lambda$ can be written in one of the forms (a) or (b).

Suppose $\lambda$ can be written as in (a). Then as in the previous paragraph, $\lambda-\alpha_{i j} \in \Lambda^{+}$. Suppose $\alpha_{r s} \nsupseteq \alpha_{i j}$ in the root poset, or in other words, suppose either $r>i$ or $s<j$ or both. One can verify that $\left\langle\lambda-\alpha_{r s}, \alpha_{s}^{\vee}\right\rangle<0$ unless $r<s=j$, in which case $\left\langle\lambda-\alpha_{r s}, \alpha_{r}^{\vee}\right\rangle<$ 0 . Thus $\lambda-\alpha_{r s}$ is not in $\Lambda^{+}$, so by Theorem $8.1, \lambda$ is join-irreducible.

Suppose $\lambda$ can be written as in (b). Then $\lambda-\alpha_{j} \in \Lambda^{+}$, and if $\alpha_{r s} \nsucceq \alpha_{j}$ in the root poset we have either $s<j$ or $r>j$. If $r \neq s$ then $\left\langle\lambda-\alpha_{r s}, \alpha_{r}\right\rangle=c_{r}-1$ and $\left\langle\lambda-\alpha_{r s}, \alpha_{s}\right\rangle=c_{s}-1$. These cannot both be nonnegative. If $r=s$ then $\left\langle\lambda-\alpha_{r s}, \alpha_{r}\right\rangle=c_{r}-2<0$, so in either case, $\lambda-\alpha_{r s} \notin \Lambda^{+}$. Thus by Theorem 8.1, $\lambda$ is join-irreducible.

\section{Chains in $\operatorname{Irr}\left(\tilde{A}_{n}\right)$}

In this section we review the standard geometric interpretation of an affine Coxeter group $\widetilde{W}$ and use it to prove Proposition 9.1, which finds the Bruhat order on the associated 
(finite) Weyl group $W$ as an interval in a miniscule quotient $\widetilde{W}^{K}$. We then organize the join-irreducibles of $\operatorname{Irr}\left(\tilde{A}_{n}\right)$ into chains, thus completing the proof of Theorem 1.1. For more details on the geometric interpretation of affine Coxeter groups, see [10].

Consider a root system $\Phi$ associated to a finite irreducible crystallographic Coxeter group $W$. For each $\alpha \in \Phi^{+}$and $k \in \mathbb{Z}$, define $H_{\alpha, k}$ to be the affine hyperplane in $\mathbb{R}^{n+1}$ consisting of all points $\lambda$ with $\langle\lambda, \alpha\rangle=k$. Define $t_{\alpha, k}$ to be the Euclidean reflection in $H_{\alpha, k}$. Then the group generated by all such $t_{\alpha, k}$ is isomorphic to an affine Coxeter group $\widetilde{W}$ with associated (finite) Weyl group $W$. Every affine Coxeter group has a presentation of this form. (Indeed, one may take this as a definition of an affine Coxeter group.) The simple generators of $\widetilde{W}$ are $t_{\alpha, 0}$ for each simple root $\alpha$ in $\Phi$ and $s_{0}:=t_{\tilde{\alpha}, 1}$, where $\tilde{\alpha}$ is the highest root in $\Phi$ (see [10]). The Weyl group $W$ is the parabolic subgroup of $\widetilde{W}$ generated by the set $\left\langle s_{0}\right\rangle$. By convention, acting on $\mathbb{R}^{n+1}$ by a simple reflection $s$ corresponds to acting on the right by $s$.

Let $\mathcal{A}$ be the collection $\left\{H_{\alpha, k}: \alpha \in \Phi^{+}, k \in \mathbb{Z}\right\}$. The set $\mathbb{R}^{n+1}-\cup \mathcal{A}$ is disconnected, and the closures of its connected components are called regions (or alcoves). We choose a base region

$$
B=\left\{\lambda \in \mathbb{R}^{n+1}: 0 \leq\langle\lambda, \alpha\rangle \leq 1 \text { for all } \alpha \in \Phi^{+}\right\} .
$$

The facets of $B$ are the hyperplanes $H_{\alpha_{i}, 0}$ for $i \in[n]$, and $H_{\tilde{\alpha}, 1}$. The vertices of $B$ are the origin and the fundamental weights $\omega_{1}, \ldots, \omega_{n}$. The group $\widetilde{W}$ acts transitively and faithfully on the set of regions, so we associate the regions to elements of $\widetilde{W}$ in a one-toone manner. Let $B$ correspond to the identity element and let $w \in \widetilde{W}$ correspond to the image of $B$ under the group element $w$. Fix $b$ to be any point in the interior of $B$. The inversion set of a region $R$ is the set of hyperplanes in $\mathcal{A}$ separating $R$ from $B$, and the length of $R$ is the cardinality of its inversion set. Recall that the weak order on a Coxeter group is containment of inversion sets and that $u \leq v$ in weak order implies $u \leq v$ in Bruhat order.

Any dominant weight $\lambda$ is a vertex of a number of regions, and among those regions, denote the region with the smallest length by $R(\lambda)$. One can find this region by drawing a straight line from $\lambda$ to $b$ and moving a small distance from $\lambda$ on that line towards $b$. More precisely, for any given finite collection of dominant weights, there is a $\epsilon>0$ such that $R(\lambda)$ contains the point $\lambda_{-}:=(1-\epsilon) \lambda+\epsilon b$ for every $\lambda$ in the collection. In what follows, we always assume that $\epsilon$ is small enough so that $\lambda_{-} \in R(\lambda)$.

With $s_{0}=t_{\tilde{\alpha}, 1}$ as above, the right quotient $\widetilde{W}^{\langle 0\rangle}$ corresponds to the set of regions $R$ such that $\left\langle\alpha_{i}, x\right\rangle>0$ for all $i \in[n]$ and $x$ in the interior of $R$. The following fact was pointed out by Stembridge [20].

Proposition 9.1. For any affine Coxeter group $\widetilde{W}$ with Weyl group $W$, the Bruhat order on the quotient $\widetilde{W}^{\langle 0\rangle}$ contains an interval isomorphic to the Bruhat order on $W$.

Proof. Let $\lambda$ be a point in the $\widetilde{W}$-orbit of the origin such that $\left\langle\alpha_{i}, \lambda\right\rangle$ is greater than the diameter of $R$ for each $i \in[n]$. (We calculate this diameter in the space spanned by $\Phi$.) Then in particular, every region containing $\lambda$ is in $\widetilde{W}^{\langle 0\rangle}$. Let $I$ denote the set of regions 
containing $\lambda$, and let $x$ be the element of $\widetilde{W}$ corresponding to the region $R(\lambda)$. Then the set $I$ is the coset $x W$, so that $I=\left\{u \in \widetilde{W}: u^{\left\langle s_{0}\right\rangle}=x\right\}$.

The affine transformation $x$ maps the set of hyperplanes of $\mathcal{A}$ containing the origin isomorphically to the set of hyperplanes of $\mathcal{A}$ containing $\lambda$. Thus since $I=x W$, for $u, v \in I$ the pair $(u, v)$ is a cover relation in the Bruhat order on $\widetilde{W}$ if and only if $\left(x^{-1} u, x^{-1} v\right)$ is a cover relation in the Bruhat order on $W$. In particular, the restriction to $I$ of Bruhat order on $\widetilde{W}$ has a unique minimal element $x$ and a unique maximal element $y$, where $y$ is represented by the region containing $\lambda_{+}:=(1+\epsilon) \lambda-\epsilon b$.

We have $I \subseteq[x, y]$ and if $u \in[x, y]$ then by Theorem 4.4(ii), $x=x^{\left\langle s_{0}\right\rangle} \leq u^{\left\langle s_{0}\right\rangle} \leq y^{\left\langle s_{0}\right\rangle}=$ $x$, so $u \in I$. We have shown that $I=[x, y]$ is the desired interval.

We now restrict our attention to the case $\widetilde{W}=\tilde{A}_{n}$, so that $W=A_{n}$. As in the previous section, we take the root system for $A_{n}$ whose positive roots are of the form $\alpha_{i j}:=e_{j+1}-e_{i}$ for $j \geq i$. The simple generators of $\tilde{A}_{n}$ described above and in Section 4 are $s_{i}=t_{\alpha_{i}, 0}$ for $i \in[n]$, and $s_{0}=t_{\tilde{\alpha}, 1}$, where $\tilde{\alpha}=\alpha_{1 n}$.

The left quotients ${ }^{\langle i\rangle} \tilde{A}_{n}$ correspond to orbits of the vertices of $B$ as we now describe. For convenience, $\omega_{0}$ denotes the origin. For $i \in[0, n]$, the quotient ${ }^{\langle i\rangle} \tilde{A}_{n}$ corresponds to the orbit of $\omega_{i}$. For each point $\lambda$ in the orbit of $\omega_{i}$, the corresponding element of ${ }^{\langle i\rangle} \tilde{A}_{n}$ is $R(\lambda)$. Each double quotient of the form ${ }^{\langle i\rangle} \tilde{A}_{n}^{\langle 0\rangle}$ is the set of dominant weights in the orbit of $\omega_{i}$.

Theorem 1.3 says that Bruhat order on each maximal double quotient ${ }^{\langle i\rangle} \tilde{A}_{n}^{\langle 0\rangle}$ is isomorphic to the standard order on the corresponding component of $\Lambda^{+}$. To prove the upper bound of Theorem 1.1, we need to construct chains which are not restricted to a single double quotient. It will, however, be possible to restrict each chain to a single maximal right quotient, which by symmetry we take to be $\tilde{A}_{n}^{\langle 0\rangle}$. We must consider the partial order on dominant weights with $\lambda \leq \mu$ if and only if $R(\lambda) \leq R(\mu)$ in Bruhat order. For the rest of the section the notation " $\leq$ " on dominant weights denotes that order, called the Bruhat order on dominant weights.

Lemma 9.2. For any dominant weight $\lambda$ and any fundamental weight $\omega_{i}$, we have $\lambda \leq$ $\lambda+\omega_{i}$ in the Bruhat order.

Proof. For any $\alpha_{j k}$,

$$
\left\langle\left(\lambda+\omega_{i}\right)_{-}, \alpha_{j k}^{\vee}\right\rangle-\left\langle\lambda_{-}, \alpha_{j k}^{\vee}\right\rangle=(1-\epsilon)\left\langle\omega_{i}, \alpha_{j k}^{\vee}\right\rangle \geq 0
$$

Thus the inversion set of $R\left(\lambda+\omega_{i}\right)$ contains the inversion set of $R(\lambda)$, so $R(\lambda) \leq R\left(\lambda+\omega_{i}\right)$ in weak order, which implies the lemma.

Lemma 9.3. If $\lambda$ is a dominant weight of the form $\omega_{i}+c_{j} \omega_{j}+c_{j+1} \omega_{j+1}+\cdots+c_{n} \omega_{n}$ for some $i<j$, then $\lambda \leq \lambda-\omega_{i}+\omega_{j}$ in the Bruhat order.

Proof. For any $\alpha_{k l}$,

$$
\left\langle\left(\lambda-\omega_{i}+\omega_{j}\right)_{-}-\lambda_{-}, \alpha_{k l}^{\vee}\right\rangle=(1-\epsilon)\left\langle-\omega_{i}+\omega_{j}, \alpha_{k l}^{\vee}\right\rangle,
$$


which is nonnegative unless $i \in[k, l]$ and $j \notin[k, l]$. However in this case we have $\left\langle\lambda, \alpha_{k l}^{\vee}\right\rangle=$ 1. Therefore $\left\langle\left(\lambda-\omega_{i}+\omega_{j}\right)_{-}, \alpha_{k l}^{\vee}\right\rangle=\epsilon\left\langle b, \alpha_{k l}^{\vee}\right\rangle$ and $\left\langle\lambda_{-}, \alpha_{k l}^{\vee}\right\rangle=1-\epsilon+\epsilon\left\langle b, \alpha_{k l}^{\vee}\right\rangle$. Since both of these are strictly between 0 and 1 , we have $\lambda \leq \lambda-\omega_{i}+\omega_{j}$ in the weak order, and therefore in the Bruhat order.

A symmetric argument proves the following lemma.

Lemma 9.4. If $\lambda$ is a dominant weight of the form $c_{1} \omega_{1}+c_{2} \omega_{2}+\cdots c_{j} \omega_{j}+\omega_{k}$ for some $j<k$, then $\lambda \leq \lambda+\omega_{j}-\omega_{k}$ in the Bruhat order.

Proposition 8.3 identifies the join-irreducibles in each component of $\Lambda^{+}$. By Theorem 1.3, each of these components is isomorphic to a double quotient ${ }^{\langle i\rangle} \tilde{A}_{n}^{\langle 0\rangle}$. By Proposition 4.6, the join-irreducibles in $\tilde{A}_{n}^{\langle 0\rangle}$ are exactly the join-irreducibles of the components of $\Lambda^{+}$. Lemmas 9.2 through 9.4 identify some order relations $\operatorname{in} \operatorname{Irr}\left(\tilde{A}_{n}^{\langle 0\rangle}\right)$. We now use these relations to decompose $\operatorname{Irr}\left(\tilde{A}_{n}^{\langle 0\rangle}\right)$ into chains. It is more tidy to write down chains in a set strictly containing $\operatorname{Irr}\left(\tilde{A}_{n}^{\langle 0\rangle}\right)$, namely the set $U$ of weights of the form $\omega_{i}+c \omega_{j}+\omega_{k}$ for $0 \leq i<j<k \leq n+1$ and $c \geq 1$. Recall that $\omega_{0}=\omega_{n+1}=0$.

For fixed $j \in[n]$ there are two ways to organize these weights into chains. One way makes a chain of the following form for each $k \in[j+1, n+1]$.

$$
\begin{aligned}
\omega_{0}+\omega_{j}+\omega_{k}<\omega_{1}+\omega_{j}+\omega_{k}<\omega_{2}+\omega_{j}+\omega_{k}<\cdots<\omega_{j-1}+\omega_{j}+\omega_{k} \\
<\omega_{0}+2 \omega_{j}+\omega_{k}<\omega_{1}+2 \omega_{j}+\omega_{k}<\omega_{2}+2 \omega_{j}+\omega_{k}<\cdots<\omega_{j-1}+2 \omega_{j}+\omega_{k} \\
\quad<\omega_{0}+3 \omega_{j}+\omega_{k}<\omega_{1}+3 \omega_{j}+\omega_{k}<\omega_{2}+3 \omega_{j}+\omega_{k}<\cdots<\omega_{j-1}+3 \omega_{j}+\omega_{k}
\end{aligned}
$$

Each element of $U$ with this fixed $j$ and $k$ appears in the chain, so every element of $U$ with this fixed $j$ is contained in one of the $n+1-j$ chains of this form.

Still keeping $j$ fixed, one can alternately create a chain of the following form for each $i \in[0, j-1]$.

$$
\begin{aligned}
& \omega_{i}+\omega_{j}+\omega_{n+1}<\omega_{i}+\omega_{j}+\omega_{n}<\omega_{i}+\omega_{j}+\omega_{n-1}<\cdots<\omega_{i}+\omega_{j}+\omega_{j+1} \\
& \quad<\omega_{i}+2 \omega_{j}+\omega_{n+1}<\omega_{i}+2 \omega_{j}+\omega_{n}<\omega_{i}+2 \omega_{j}+\omega_{n-1}<\cdots<\omega_{i}+2 \omega_{j}+\omega_{j+1} \\
& \quad<\omega_{i}+3 \omega_{j}+\omega_{n+1}<\omega_{i}+3 \omega_{j}+\omega_{n}<\omega_{i}+3 \omega_{j}+\omega_{n-1}<\cdots<\omega_{i}+3 \omega_{j}+\omega_{j+1}
\end{aligned}
$$

Again, each element of $U$ with this fixed $j$ is contained in one of the $j$ chains of this form.

For each $j \in[n]$ we choose whichever method gives the fewest chains for a total of

$$
\sum_{j=1}^{n} \min (n+1-j, j)=\left\lfloor\frac{(n+1)^{2}}{4}\right\rfloor
$$

chains covering $\operatorname{Irr}\left({ }^{\langle 0\rangle} \tilde{A}_{n}\right)$. By symmetry and Proposition 4.6 we cover $\operatorname{Irr}\left(\tilde{A}_{n}\right)$ by $n+1$ times this many chains, thus proving the upper bound of Theorem 1.1. 
Remark 9.5. Similar reasoning to Lemmas 9.2 and 9.3 shows that for $1 \leq i<j \leq n$, the weight $\omega_{i}+\omega_{j}$ is a dissector of its component of $\Lambda^{+}$. However, using this description of the dissectors to prove the lower bound of Theorem 1.1 would be difficult because one needs to construct an antichain in $\operatorname{Dis}\left(\tilde{A}_{n}\right)$, rather than one antichain in each $\operatorname{Dis}\left(\langle i\rangle \tilde{A}_{n}\right)$.

\section{Acknowledgments}

The authors wish to thank Vic Reiner and John Stembridge for helpful conversations.

\section{References}

[1] S. Billey, W. Jockusch, and R. Stanley, Some combinatorial properties of Schubert polynomials, J. Algebraic Combin. 2 (1993) no. 4, 345-374.

[2] A. Björner and F. Brenti, Affine permutations of type A., The Foata Festschrift, Electron. J. Combin. 3 (1996) no. 2, Research Paper 18.

[3] A. Björner and F. Brenti, Combinatorics of Coxeter Groups, Graduate Texts in Mathematics, Springer Verlag, to appear.

[4] N. Bourbaki, Lie groups and Lie algebras. Chapters 4-6. Translated from the 1968 French original by Andrew Pressley. Elements of Mathematics. Springer-Verlag, Berlin, 2002.

[5] I. Chajda and V. Snášel, Congruences in Ordered Sets, Math. Bohem. 123 (1998) no. $1,95-100$.

[6] V. Deodhar, A splitting criterion for the Bruhat orderings on Coxeter groups. Comm. Algebra 15 (1987), no. 9, 1889-1894.

[7] H. Eriksson, Computational and Combinatorial Aspects of Coxeter Groups, Ph. D. thesis, KTH, Stockholm, Sweden, 1994.

[8] K. Fan, On Dilworth's Coding Theorem, Math Z. 127 (1972) 92-94.

[9] M. Geck and S. Kim, Bases for the Bruhat-Chevalley Order on All Finite Coxeter Groups, J. Algebra 197 (1997) no. 1, 278-310.

[10] J. Humphreys, Reflection Groups and Coxeter Groups, Cambridge Studies in Advanced Mathematics, 29, Cambridge Univ. Press 1990.

[11] A. Lascoux and M.-P. Schützenberger, Treillis et bases des groupes de Coxeter, Electron. J. Combin. 3 (1996) no. 2, Research Paper 27.

[12] G. Lusztig, Some examples of square integrable representations of semisimple p-adic groups, Trans. Amer. Math. Soc. 277 (1983) no. 2, 623-653. 
[13] G. Markowsky, Primes, Irreducibles and Extremal Lattices, Order 9 (1992) no. 3, 265-290.

[14] N. Reading, Order Dimension, Strong Bruhat Order and Lattice Properties for Posets, Order 19 (2002) no. 1, 73-100.

[15] N. Reading, Lattice and Order Properties of the Poset of Regions in a Hyperplane Arrangement, Algebra Universalis 50 (2003), 179-205.

[16] R. Stanley, Enumerative Combinatorics, Volume I, Cambridge Studies in Advanced Mathematics, 49, Cambridge Univ. Press 1997.

[17] J. Stembridge, On the fully commutative elements of Coxeter groups, J. Algebraic Combin. 5 (1996) no. 4, 353-385.

[18] J. Stembridge, The partial order of dominant weights, Adv. Math. 136 (1998) no. 2, $340-364$.

[19] J. Stembridge, Tight quotients and double quotients in the Bruhat order, Electron. J. Combin. 11(2) (2005), R14.

[20] J. Stembridge, personal communication, 2004.

[21] W. Trotter, Combinatorics and Partially Ordered Sets: Dimension Theory, Johns Hopkins Series in the Mathematical Sciences, The Johns Hopkins Univ. Press 1992. 